\title{
A Taxonomy for Visualisations of Personal Physical Activity Data on Self-Tracking Devices and their Applications
}

\author{
Majedah Alrehiely \\ Parisa Eslambolchilar \\ Rita Borgo \\ School of Computer Science and School of Computer Science and Informatics Department \\ Informatics, Cardiff University, UK Informatics, Cardiff University, UK Kings College London, UK \\ AlrehielyMM@cardiff.ac.uk \\ EslambolchilarP@cardiff.ac.uk \\ rita.borgo@kcl.ac.uk
}

\begin{abstract}
Self-tracking devices and apps have been widely used for personal data collection, with particular focus on health and physical activity (PA) monitoring. Despite their pervasive use, data representation and data sharing on these devices and apps are still in their infancy. With the aim of contributing towards structuring the design space of personal health visualisation, we present an overview focused on visualisation methods and the typology of tracked data in the most popular health and PA tracking devices and their companion apps/dashboards. Our research method of data collection is based not only on a review of scientific literature in the field, but also on autoethnography, information collected from manufacturers' websites and user manuals as well as online communities and reviews. We then discuss the major issues and limitations users face with regards to health and PA data interpretation and sharing.
\end{abstract}

Smartphone Apps, Wearable Devices, Health Tracking, Smartwatches Self-tracking, Personal Visualization.

\section{INTRODUCTION}

Personal tracking is not a new subject; people have been tracking their own activities using different methods, such as diaries, for many centuries and personal computers for many decades (Rooksby et al., 2014). However, self-tracking has become more popular with the advancement of smartphones and wearable devices, which have embedded accelerometers and built-in GPS for activity tracking indoors and outdoors (Rooksby et al., 2014).

With more sophisticated embedded sensors and data classification algorithms becoming available on smartphones and smartwatches, the concept of personal tracking is expanding rapidly to capture different types of data about oneself covering different domains. For example, nowadays, it is possible to record data on leisure and sport activity (e.g. Fitbit, MapMyWalk), calorie intake (e.g. MyFitnessPal), liquid consumption (e.g. Waterlogged), locations (e.g. Moves), phone usage (e.g. Moment) and many others. Given the large spread and variety of information, we chose to focus our work on one specific, but very important aspect of personal data, namely health data.

According to a study conducted by "Research 2 Guidance" health research group in 2016 (Reseach2Guidance, 2016), the number of mobile health or mHealth apps available in the major app stores in 2016 was around $259 \mathrm{~K}$, with a total download rate of 3.2B. This study also reported a significant increase in the growth of sales of health and fitness tracking devices. Health apps and tracking devices often collect different health metrics, therefore they generate large collections of heterogeneous health data. These large volumes of collected data can potentially increase the difficulty of integration, understanding and sense-making of personal health data across different platforms (Mayer et al., 2016).

Visual encoding of information has always played a major role in communication and, when used appropriately, it can become a powerful tool to guarantee access to information, especially for lay users. Visualisation of tracked health data from both an aesthetic point of view and a user informative point of view, is a very important aspect of both mHealth apps and wearable devices' companion software. Although most self-tracking devices offer some data visualisations, some self-trackers faced difficulties in terms of data interpretation and built their own customized visualisation tool as discussed in (Choe et al, 2014).

This article aims to build a bridge between published research, which explores the user experiences of self-tracking devices and the information collected from manufacturers' websites, user manuals and online communities and Weblogs with respect to personal health data visualisation. We build on previous studies that focus on the reflection stage of 
personal informatics model (Li et al., 2010), which are (Li et al., 2011) and (Choe et al., 2014), to discuss the visualisations. We present an up-to-date overview of the data representation methods provided by companion apps and embedded displays of four self-tracking devices and discuss their limitations and strengths based on self-trackers' requirements and self-reflection's needs. This is a preliminary step that could contribute in structuring the design space of personal health visualisation and improving the current visualisation designs.

The structure of the paper is as follows. We start with a background on personal health tracking and visualisation followed by a discussion of our research methodology. We then discuss the basic visualisation layouts provided by each device and companion apps. We conclude with a discussion on the issues of visual representation of the data on different tracking devices and their companion apps. Finally, we provide some suggestions to address the issues highlighted.

\section{BACKGROUND AND RELATED WORK}

The field of personal health is broad and overlaps with many fields, such as mobile Health (mHealth) and $\mathrm{HCl}$. In recent years, researchers have explored personal health tracking from different perspectives. In this section, we reflect on and discuss the related literature from two research areas: personal informatics and personal health visualisation.

\subsection{Data Visualisation in Personal Informatics}

Personal informatics is defined as the process of gathering and reflecting on personal data to gain better self-knowledge ( $\mathrm{Li}$ et al., 2011). Li et al. (2010), developed a five stage-based model of personal informatics, which are preparation, collection, integration, reflection, and action and they listed the barriers people face in each stage. This model was derived from surveys and interviews with users of different personal informatics tools that studied their practice, motivations and issues in each stage. They identified some problems users faced with the visualisation of their data during the reflection stage, which affects transition to the action stage, such as failing to use visualisations effectively to extract knowledge from the data. Li et al. noted the importance of referring to different fields (e.g. information visualisation) to resolve issues within each stage (Li et al., 2010).

Since developing effective tools for supporting selfreflection requires sufficient understanding of people's self-reflection needs, Li et al. (2011) built on their previous study (Li et al., 2010) and interviewed 15 people who used different types of selfinformatics tools to explore and identify the questions people have on their personal data. They also discussed what tool the participants' used to reflect on their data, how they used it and what issues they encountered. Their study resulted in identifying six types of questions that people usually ask about their personal data during two non-static phases of reflection i.e. discovery and maintenance. Those six types of questions relate to status, history, goals, discrepancies, context, and factors. Although the authors discussed how the participants used their tools to reflect on the data and to answer their questions, there were no details on the visualisation methods used and their limitations.

Choe et al. (2014) focused on the reflection stage and they analysed 52 video recordings of Quantified-Selfers talks in Quantified-Self meetings with the goal of understanding the practice of selftrackers for improving the designs of self-informatics tools. Their study explored important aspects of selfreflection by analysing how the Quantified-Selfers analysed their data and what visualisation they used (Choe et al., 2014). In follow up research (Choe et al., 2015), Choe et al. analysed 30 recorded videos of Quantified-Selfers presentations to identify types of insight and what visualisation method the presenters used to convey their insight. They identified 8 data-driven types of visualisation insights (detail, self-reflection, trend, comparison, correlation data summary, distribution and outlier).

A survey by Huang et al. (2015), provided an extensive survey for personal visualisation systems that support personal data analysis for nonprofessional users. The survey explored the design space and highlighted key challenges in the field including the adoption of personal visualisation and personal visual analytics technologies, sharing and privacy, and evaluation (Huang et al., 2015).

Our research differs from the previous data visualisation studies in personal informatics in two ways: firstly, we explore the visualisation methods that are available on the most popular tracking devices and applications with a particular focus on health and PA data. secondly, we examine whether the current visualisations supported by such devices meet the users' needs derived from the previous studies and report on what is missing in personal health visualisation design space.

\subsection{The Visualisation of Personal Health Data}

The availability of health data generated by selftracking devices has started to increase patients' awareness and sense of responsibility regarding their health conditions (Shneiderman et al., 2013). These devices (e.g. Fitbit) also support visual representations of the tracked data which can be viewed on their companion apps and/or online dashboards, and in some devices, the visualisation could be displayed on the device screen as well. The visualisation methods used to transform the data into an easy-to-understand format are 
represented in traditional chart formats, such as bar chart and line chart. Although these charts provide awareness and allow searching for specific information and details (Fan et al., 2012), they have some limitations and they need to be evaluated within the context of the personal health domain. One example of such an evaluation is the study carried out by (Tong et al., 2015) who evaluated a Fitbit bar chart with circular ringmap and Virtual Pet visualisation. The evaluation was conducted taking into account five factors: Readability, Comprehension, Awareness, Attractiveness and Empathy. In their study, Tong et al. (2015) showed that efficiency of the visualisation is unrelated to the participants' subjective evaluation and that each visualisation method provides a different view of the data. Their results also shed light on the significance of having new visualisation designs that serve these factors.

Different research projects studied the visualisation of personal health data and developed different visualisation designs for personal health and PA tracking data to support reflection. Epstein et al., (2014), investigated the motivations for self-tracking and examined a new approach to help people (selftrackers) discover valuable and actionable correlations in their data. They defined a set of cuts (i.e. selected subset of tracked data that shares some related features) over location and PA data, and used different visualisations to represent these cuts. Meyer et al. (2016), discussed the challenges of visualising heterogeneous health data on the small display of mobile devices. They developed and evaluated different designs of both metaphoric and quantitative visualisations to provide an overview and details on demand for different health data. They proposed different types of visualisation to support short-term and long-term representation (e.g. tree metaphor, bar and pie charts and bubble graphs). Their findings showed that people are interested in finding more complex correlations in their health data that could not be configured using simple methods of data representation (Mayer et al., 2016).

Other research focused more on developing abstract representations, such as UbiFit (Consolvo et al., 2008), which used a garden metaphor to represent different types of PA data and Fish ' $n$ ' Steps (Lin et al., 2006) that linked each user's step count to the growth and activity of a single virtual fish. These living metaphors were developed to encourage the users to be more active, as the users became emotionally attached to these metaphors. Emotional engagement is an important factor in personal visualisation that promotes personal motivation toward lifestyle improvement (Tong et al., 2015). However, these designs could also allow users develop negative emotions, such as guilt, as reported in Lin et al. (2006) study.

Good visualisation is a key element in personal health technology design space (Bardram \& Frost, 2016). Although there have been many research articles discussing the visualisation of personal health data, there has not been sufficient discussion or research on the existing visualisations provided by commercial health tracking devices and their companion apps.

In this paper, we explore the visualisation methods provided by four popular self-tracking devices and their companion apps in the context of personal health tracking. We believe that discussing issues and limitations of such devices with regards to data visualisation, interpretation, reflection and sharing, our research may provide some insight regarding how to represent health and physical activity data. We provide an updated view of the typology of the automatically tracked data, how these data are presented on the different platforms and what level of view each visualisation provides. We have considered health data that are automatically tracked by self-tracking devices (i.e. PA, sleep and heart rate) in discussing the visualisation layouts; health data manually entered, such as nutrition, smoking and alcohol intake are not included in this article.

\section{RESEARCH METHOD}

\subsection{Device Selection}

We have shortlisted and selected self-tracking devices reviewed in this paper based on two sources: the IDC report on unit shipment and market share of wearable vendors in Q4 2016 (IDC Research Inc, 2017) and the results from our online survey that was conducted to understand PA tracking and the devices used in addition to trackers' preferences in visualising their data.

Our online survey was conducted in the period between November and December 2016 to study users' preference in PA tracking generally and in visualisation and sharing data specifically. The survey was circulated among different mailing lists, and to people who were interested in health and activity and self-tracking (such as Quantified Self forum and Sports Union mailing list at Swansea University). 84 participants responded to the survey, of whom $89 \%$ specified what devices they used for tracking while around $11 \%$ did not use any tracking device. The results showed that the most used tracking devices in order were: Fitbit 38\%, Apple watch $13 \%$, Garmin 7\%, iPhone 7\%, Android phones $7 \%$ and Jawbone and Withings at $4 \%$ each. 
Table 1: Steps taken for selecting the tracking devices to review in this article.

\begin{tabular}{|c|c|}
\hline $\begin{array}{l}\text { Selection } \\
\text { Criteria }\end{array}$ & $\begin{array}{l}\text { (1) Choosing } 4 \text { of the most popular activity tracking devices based on IDC (IDC Research, Inc, 2017) } \\
\text { and the results for the most used tracking devices by the participants in our online survey } \\
\text { (2) From these vendors, we are selecting the most recent trackers that have a large display and track } \\
\text { more than two PA data. The chosen devices are as follows: }\end{array}$ \\
\hline \multicolumn{2}{|r|}{ Smartwatches } \\
\hline Brand & Version \\
\hline Fitbit.com & $\begin{array}{l}\rightarrow \text { Products } \rightarrow \text { Smart Fitness Watches } \rightarrow \text { Two are available: Surge (released in early } 2015 \text { (Fitbit, } \\
2014) \text { ) and Blaze (released in } 2016 \text { (Business Wire, 2016; Fitbit, 2016a) } \rightarrow \text { Fitbit Blaze }\end{array}$ \\
\hline Apple.com & $\begin{array}{l}\rightarrow \text { Watch } \rightarrow \text { Series } 1 \text { and Series } 2 \rightarrow \text { Series } 2 \text { ( } 4 \text { models: Series } 2, \text { Nike+, Edition and Hermes) } \rightarrow \text { by } \\
\text { comparing the } 4 \text { models on Apple.com, they are all series } 2 \text { with the same operating system, tracking } \\
\text { capabilities and visualisation and differ in the design } \rightarrow \text { Apple Watch Series } 2\end{array}$ \\
\hline Garmin.com & $\begin{array}{l}\rightarrow \text { Shop } \rightarrow \text { Wearable: Activity trackers } \& \text { watches } \rightarrow \text { The latest according to (Garmin Press Release, } \\
\text { 2017a, 2017b) are: Forerunner } \circledast 935 \text { (GPS watch and activity tracker) and VivoSmart } 3 \text { (no GPS } \\
\text { activity tracker and has a smaller display) } \rightarrow \text { Forerunner }{ }^{\circledR} 935\end{array}$ \\
\hline Samsung.com & $\begin{array}{l}\rightarrow \text { Mobile } \rightarrow \text { Wearable } \rightarrow \text { Smartwatches } \rightarrow \text { Gear (S2/ S2 Classic) and Gear S3 (Classic/ Frontier) } \rightarrow \\
\text { Gear S3 comes in two designs: frontier and classic to fit different lifestyles (Samsung.com, 2016), they } \\
\text { share the same features and system } \rightarrow \text { our review will be about Samsung Gear S3 in general }\end{array}$ \\
\hline
\end{tabular}

The "IDC Worldwide Quarterly Wearable Device Tracker" report published on March 2, 2017 (IDC Research, Inc, 2017), provided valuable information on the worldwide popularity and sales numbers of the devices. In this report, the top 5 wearable devices in Q4 of 2016 based on unit shipments and market share were ordered as Fitbit, Xiaomi, Apple, Garmin, and Samsung.

Based on our Online Survey and Wearable Device Tracker's market (IDC Research, Inc, 2017), we shortlisted Fitbit, Apple watch and Garmin as they had the highest popularity. In addition to these three trackers, we shortlisted Samsung as it is the fifth top wearable devices vendor. We excluded mobile phones as the focus of the research was on wearable trackers and we also excluded Xiaomi because the brand was not widely recognised outside the country it was being manufactured (IDC Research, Inc, 2017).

As we intend to review the visualisation displayed on the device screen in addition to the visualisations displayed on their companion app interfaces and online dashboards, we decided to choose the latest version of the devices that have larger displays i.e. smart watches from our shortlisted vendors, in order to review devices with identical features. Therefore, our final devices are Fitbit Blaze, Garmin Forerunner 935, Samsung Gear 3 and Apple Watch series 2; we have not included activity trackers in our analysis. Table 1 presents our method for shortlisting and device selection.

\subsection{Device Review}

Given the rapid development of tracking technology and the human-centred focus of our work, we chose to enrich the traditional revision of scientific and technical literature with contributions from devices' official websites and user manuals, users' online communities and forums in addition to some online users' reviews for some devices and apps. In reviewing Fitbit Blaze data visualisation features, we relied on its User's Manual (Fitbit, n.d.-b), Introductory Guide (Fitbit, n.d.-a) and videos from Fitbit YouTube Channel (Fitbit, n.d.-c) (e.g. (Fitbit, $2016 b)$ ). For reviewing the visualisation on the app and on the dashboard, we extended our search to include more general sources on the Fitbit, which are related to the Fitbit trackers in general; for example all Fitbit trackers synchronize to the same companion app and the dashboard webpage (Fitbit, 2017). For reviewing visualisation on Garmin Forerunner 935 display and its companion app and dashboard, we relied on its User's Manual (Garmin, n.d.-a) and the related videos on Garmin YouTube channel (Garmin, n.d.-b). Due to lack of screenshots of the device display and the graphs of the data, we included additional sources that reviewed the watch e.g. (DC RAINMAKER, 2017; Michael Sawh, 2017). Moreover, to obtain more detail about the visualisation on Garmin Connect app and dashboard, we included the reviews of other versions of Garmin forerunner e.g. (GadFit, 2015) and (Luke Edwards, 2016). For reviewing Samsung Gear S3 and Samsung Health app (Samsung Electronics Co., Ltd., 2017), we referred to Gear S3 User's Manual (Samsung, 2017), related videos on Samsung YouTube Channel (Samsung, n.d.) (e.g. (Samsung, 2016)), different articles about Samsung Health app and Gear S3 posted on Samsung newsroom (Samsung Newsroom, 2015, 2016a, $2016 \mathrm{~b}, 2016 \mathrm{c}, 2017)$ and other sources that reviewed the watch, such as (Techisode TV, 2017) and (Analytical, 2016) in addition to our personal experience with using Samsung health app itself without the watch.

Moreover, we used autoethnography (Cunningham \& Jones, 2005) in cases where we owned a device or when we had access to an app or a device's 
companion dashboard. We used autoethnography to strengthen our reviews of Apple Watch, iOS Health App, iOS Activity App, Fitbit app on an iOS platform and Fitbit Online dashboard (without Fitbit device), Garmin Connect app and dashboard and Samsung Health App (the basic app without using the watch) in addition to the sources listed on each device review that will be presented in the following sections. Visual layout choices within Fitbit Community are highly dependent on the type of data collected by the specific device.

To ensure an unbiased review, we extracted different details from the varied selected data sources (including screenshots and videos) about how the same tracked data are actually presented across the devices and their platforms, personal opinions or experiences were not considered.

\subsection{Analysis Method}

After selecting the devices and collecting the data sources for reviewing the visualisations, we created a profile for each device by capturing different information about the data and how they are presented. Firstly, we classified the visualisation methods based on the platform(s) that support any sort of visualisation for the data tracked by the device (e.g. the device's screen, Online dashboard). Although all shortlisted devices in this article track similar activity data (e.g. step count, distance, flights climbed, heart rate (HR) and calories burned in addition to exercise detail such as running and cycling), they vary significantly in the way they visually represent the activity data. Therefore, for each platform, we captured the following information: (1) what data are visualised, (2) how different PA data are presented, and what visualisation method(s) are used and, (3) the length of data being visualised: current/recent data visualisation versus history/past data visualisation. Lastly, we summarized and categorized the collected information using a multi-dimensional table (Table 4 within the appendices). The different dimensions present different aspects about the data visualisation methods.

\section{DATA VISUALIZATION METHODS ON SELECTED DEVICES}

Table 4 summarizes the data visualisation methods used in representing data collected by each device. The abbreviations for the visualisation methods are defined in

Table 2. The colour codings we have used in Table 4 are defined in Error! Reference source not found. and refer to the length of time of the data being visualised (see Table 2, Table 3Table 4 within the appendices).
We identified several common representation methods across the different platforms, such as the widely used traditional bar and line charts. The visualisation methods can be grouped into five major categories: chart based, radial based, calendar based, metaphorical and highdimensional.

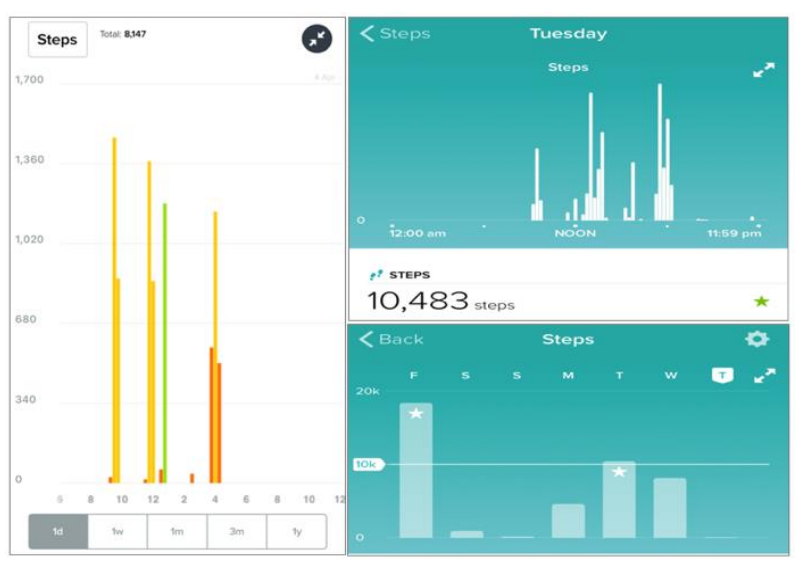

Figure 1 Three screenshots from Fitbit app. Left and top: show detailed bar chart for steps during a day. Bottom screenshot shows bar chart for steps of seven days. The star on the bars means that the user met the steps goal for that day ".

\subsection{Chart}

Chart based layouts are common across all devices and companion apps that we reviewed, and differences appear mostly at the level of display size and resolution.

As shown in Table 4, the Fitbit app and dashboard provide different options for representing the data for the current day versus historical data. The "overview" of the data is mainly presented via bar charts based on days, and a detailed bar chart can be viewed by selecting a specific day. Figure 1 and Figure 2 are screenshots of Fitbit app viewing different visualisations for different data. Fitbit provides a coloured line chart for HR data for a single exercise where the colours refer to the HR zones to show how the user is performing during the exercise, in addition to a bar chart to represent the time in HR Zones (as seen in (Fitbit, n.d.-a, 2016b)).

Bar chart and line chart are the preferred visual layouts of Apple Health app (Apple Inc, 2017) when dealing with daily data at high resolution e.g. hourly based, or to provide a historical overview e.g. week vs. month vs. year.

The graphs and statistical charts provided by Garmin are more focused on exercises rather than viewing a historical overview of users' activity level over time. It offers a number of detailed graphs for physical exercises that are not available on the companion apps of other devices. For example, line chart

${ }^{*}$ All the images used in this paper belong to the authors 
representing HR information for a single day PA (see Figure 7) has colour coding that represents time in $\mathrm{HR}$ zones in addition to the glyphs on the time axis that show the type of exercise that has been performed and the sleep time. It also represents HR data in different graphs (Bar Chart/Line Chart) that show time in HR zones, even for a single exercise.

Samsung Gear S3 employs a bar chart to display step count history where data collected in the past seven days can be viewed at one time. On the Samsung Health app, the historical data can be viewed on the trends section. The bar chart visualises one-week of data (e.g. active minutes). Although the user can view past as well as current data, it displays only seven days of data at one time on the screen.

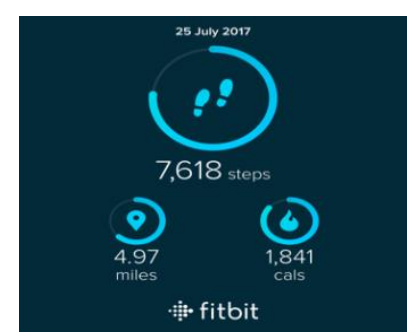

Figure 2 Fitbit circular rings for steps, distance and calories burned in a day.

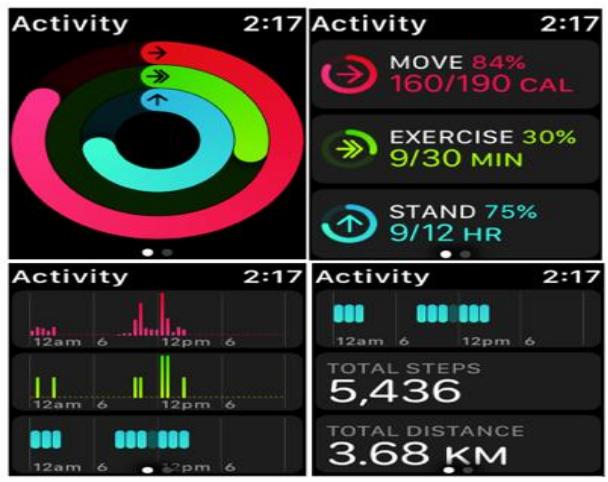

Figure 3 Four screenshots captured from Activity app running on Apple watch. Top left shows the circular ring that represents active calories, exercise minutes and standing hours, followed by more details on the activities.

\subsection{Radial Projection}

A circular ring that moves towards a daily activity goal provides its users with quick feedback on their activity progress throughout the day. On the Fitbit app, the user is also able to view the visualisation of different data from one day up to one year.

On the Fitbit Blaze display, the user can see Circular Rings of the current day's data, such as step count and distance, and these circular rings are also viewed on the Fitbit app/dashboard (see Figure 2). The visualisation of activity data tracked by Apple watch is mainly provided by two apps: Health app (Apple Inc, 2017) and Activity app (Apple Support, 2017). The latter is related to the watch itself; there is no online dashboard or desktop application, so the view is limited to the phone and the screen of the watch. The visualisation on the watch display is a Circular Ring that combines three different variables with different colours (see Figure 3). The red ring represents active calories burned, the green represents exercise duration in minutes and the blue represents standing hours.

\subsection{Calendar}

The Activity app on the iPhone shows Circular Rings on a calendar layout (see Figure 4). The user can interact with the calendar to view the detailed bar chart for each day. The Health app also uses the calendar as the main layout to view the information but there is no visualisation combined with the calendar view; the user can view the details by selecting a specific day on the calendar. Garmin and Fitbit apps use calendar view to show logged exercises. These calendars are interactive, and additional details about logged exercises can be viewed on demand.

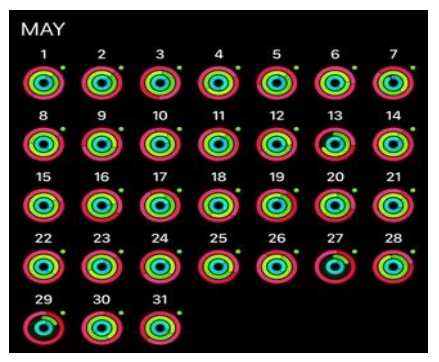

Figure 4 A circular ring visualisation of one-month activity data - a screenshot from iOS Activity app.

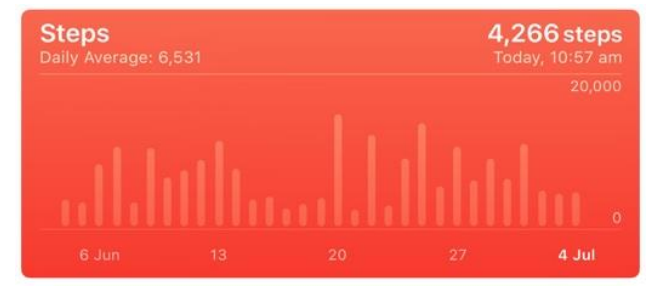

Figure $5 \mathrm{~A}$ bar chart visualisation of one-month activity data - a screenshot from the iOS Health app.

\subsection{Metaphorical}

Rich visual metaphors, such as icons, are a very powerful tool to convey multidimensional information; moreover, when the metaphor features a familiar link with the user, cognition and information retention are increased (Borgo et al., 2012).

Samsung offers a distinctive visualisation on the display of Gear S3, which uses a clock metaphor to represent the minutes of the user's activity during the day, along with sleep hours (as presented in (Analytical, 2016; Samsung Newsroom, 2016c)). Colour coding is used to distinguish between sleep hours, minutes of healthy and light activity and the time when the user was not active. The user can interact with the visualisation to view further details, such as calories burned. This visualisation could provide the user with a quick view of the level of activity and sleep for the current day. The data 
visualised on the watch is not restricted to the current day's data only; the user can access the data from previous days.

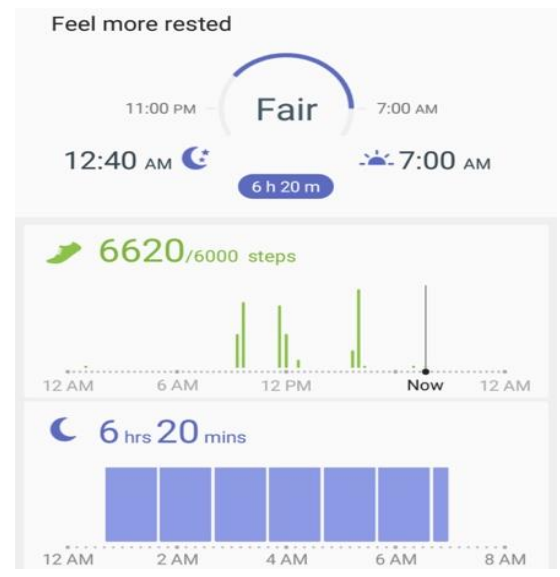

Figure 6 A Screenshot of Samsung Health app. On the app interface, a bar chart represents step count of the current day sleep.

\subsection{High-dimensional}

The Activity app provides details about different workouts, but the details are mainly presented in textual format. However, it presents a route on a map to visualise outdoor workouts, such as an outdoor run. Furthermore, Activity app is more focused on PA data while Health app includes the details and the visualisation of other health data, such as sleep and nutrition. Although there are some useful visualisations for the data tracked by Apple watch, such as the calendar view provided by Activity app, there are some limitations such as the absence of HR visualisation. Garmin Connect dashboard provides an animated visualisation for exercises (e.g. running or cycling) that simulates the user activity. It presents the route on the map along with a line chart for representing the speed and elevation during the exercise and coloured bars for representing all data metrics that are changed based on the movement on the map, such as total time and $\mathrm{HR}$, as presented in (Garmin, 2009).

Text and numerical representation of data is common to all devices. On Fitbit Blaze, the user is able to view details of different workouts in text and number format, as well return to either app or dashboard for more detailed charts integrating textual and numerical information. The Activity app also provides details about different workouts, but the details are mainly presented in textual formats.

\section{DISCUSSION}

Visual representation methods play a significant role in reducing the cognitive load of data interpretation for users and enabling users to easily understand large data (Le et al., 2013). Personal health data could be of interest not only to the person himself but to others such as health providers, patients groups and family members (Le et al., 2013). In the following sections we discuss the limitations of the data representation methods on the reviewed devices and the level of control the users have on their data with regards to sharing health data.

\subsection{Visual Layout}

To facilitate the discussion about the visualisation layouts, we classify the visualisations into categories based on their designs. And then discuss the main challenges in visualising personal health data.

\subsubsection{Detailed View of Short-Term Data}

Reflection on short-term data is valuable for making users aware of their status (Li et al., 2010), to see if they are meeting their goal and whether they should take an action toward their behaviour ( $\mathrm{Li}$ et al., 2011).

All reviewed devices and companion apps in this article support short-term data visualisation using different methods such as circular ring and text. Detailed view of the data is provided either for a day or for a physical activity (e.g. a bar chart representing step counts throughout the day), in addition to an overview that provides the user with a quick feedback on the progress made towards a goal (e.g. a circular ring representing the progress made on being active, sitting less or calories burned and their respective targets).

For exercise visualisations, reviewed devices in this article provide different types of visualisation as discussed earlier. Fitbit app, as an example, provides a detailed line chart for visualising HR data during an exercise as (see (Fitbit, n.d.-a)). Garmin supports detailed representation of different variables of exercises. As an example, for running workouts, it shows statistical charts for pace, elevation and HR individually or overlapping on a time axis (see Figure 8). It also represents the duration of running in addition to the route of the run on the map. Garmin dashboard provides an animated visualisation for exercises where each graph represents one data variable only (see (Garmin, 2009)). Combining the different graphs into one visualisation (shared space) could be more efficient for comparison across visual representations or the same or similar data since it would not require the user's gaze to move across different graphs to assess differences or similarities (Javed et al., 2010).

Another limitation in the visualisation designs for short-term data appears in the lack of ability to personalize the visualisations to suit users' tracking purposes. For example, the main visualisation for current data (status) on Apple watch and Activity app is designed for goal driven tracking, which is the circular rings that move toward goal completion. This is not an optimal visualisation design for all users as 
they vary significantly in their tracking purposes according to (Li et al., 2011; Rooksby et al., 2014). Moreover, users have no control over what variables to present in the visualisation. Although some platforms support customization, such as Fitbit app and dashboard, these customization options are not directly applied to the visualisation itself and they only enable adding or removing some data that are presented as separated widgets on the interface.

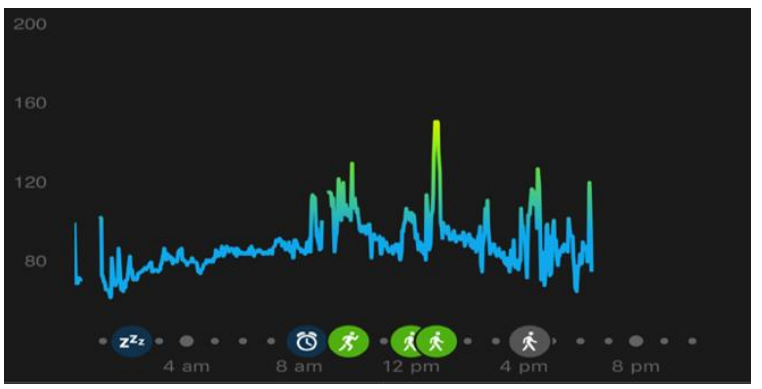

Figure 7 A screenshot of Garmin Connect HR Details for a single day.
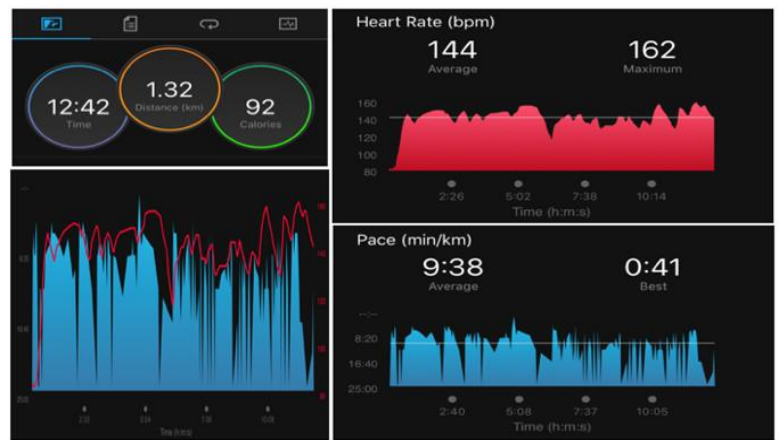

Figure 8 Three screenshots from Garmin Connect app showing different data charts for a running exercise.

\subsubsection{Overview of Long-Term Data}

A lack of interest in personal historical data has been identified by many researchers, such as (Gouveia et al., 2015; Rooksby et al., 2014). A potential reason for people being less interested in their historical data could be the difficulties of comprehending and making sense of the collected data, especially as tracked data grows massively and becomes complex over time. Data interpretation difficulties were reported by many Quantified-Selfers in Choe et al. (2014)'s study which also observed that many people tried to simplify their tracking strategy after they failed in reflecting on their data.

Reflection on long-term data can reveal valuable information and finding patterns and trends ( $\mathrm{Li}$ et al., 2010). In reviewed devices, statistical charts are the most common methods used to represent historical data, as presented in Table 4. A study (Asimakopoulos et al., 2017) conducted with 34 Jawbone and Fitbit trackers users showed that the data visualisation designed for these trackers' companion apps appears to be useful for the viewing of short-term data and tends to be less useful for the viewing of historical data. The participants in the study by Tong et al (2015) reported difficulties in identifying PA patterns when using Fitbit bar chart, while this task was easier with circular ring visualisation. Tong et al. suggested implementing interaction features for Fitbit bar chart to allow faster data exploration and patterns recognition. Moreover, in the study by Meyer et al. (2016), it was indicated that users are interested in discovering more complex correlations in their health data that could not be identified using simple visualisation methods over both short and long timeframes. This shows a necessity for more advanced methods for representing and analysing long-term data as they are mainly visualised with the statistical charts.

Apple Activity app offers a different form of representation for long-term data using circular ring visualisation on a calendar view (presented in Figure 4). A main disadvantage of this method in visualising long-term data is that full rings are not always reflecting how the users are progressing in their activity as the users could change their goal settings over time. However, the user could identify each day view by clicking on the circular ring to obtain details.

\subsubsection{Supporting Multiple Data Visualization}

As discussed earlier, two out of five questions people ask about their self-tracked data are related to context and factors ( $\mathrm{Li}$ et al., 2011). The context is to seek what could affect the present data while factors seek what influence a personal behaviour has over the long-term ( $\mathrm{Li}$ et al., 2011). Finding answers to these two questions requires identifying correlations between different data and this depends on users' ability to access additional data (Li et al., 2011). For example, a person with a weight loss goal may need to see how other factors, such as increasing PA levels, affect weight. Here, we discuss multiple data visualisation for context and factor.

Effective multiple data visualisation requires users to be engaged with the visualisation to customize data selection. In the study by Meyer et al., different forms of visualisations were evaluated for heterogeneous health data (step count, calories burned, weight and sleep) on mobile application, and the users showed interest in the development of integrated visualisations. The participants requested supporting analytical features in the visualisation, such as data filtering, for more effective health reflection that could identify complex relations in the data. Our review found that long-term data, generally visualised using statistical charts, mostly represents data for one variable only (e.g. step count) and those charts do not visualise combined multiple activity variables. Multiple data visualisation is found on Apple Activity app and on Apple watch screen (Figure 4). This goal-driven design is composed of three circular rings, where each ring refers to a different variable. 
Multiple data visualisation for short-term data (i.e. context) was more supported by reviewed devices and apps than long-term data. A good example of multiple data visualisation for short-term data could be found in Garmin HR Details (Figure 7), which combines HR levels with user's activity modes e.g. running or sleeping. Another example would be Samsung watch display that uses a clock metaphor to represent sleeping hours combined with minutes of activity throughout the day. Moreover, in calendar view in Apple Activity app, the circular ring visualisation for each day displays three variables at a time: active calories, exercise minutes and standing hours. Therefore, circular ring also provides an overview of historical data at a glance.

The limitations in these reviewed visualisations appear in supporting appropriate visualisation designs for long-term multiple data and in supporting additional interaction features, such as allowing users to choose the length of data and variables to be visualised. Providing such features may help the users in generating and testing hypotheses about their personal behaviour which could reveal information such as causation and correlations as noted in (Choe et al., 2014).

For visualising multiple personal health data, the visualised data must not be restricted to health data variables only as many other non-health-related factors could affect personal health. For example, (Huang et al., 2016) investigated the influence of using calendar's data (e.g. events) to provide extra context for users to help them reason regarding their fitness. They developed a tool that integrates a quantitative visualisation of PA data with a personal calendar. The results from this study confirmed that personal calendars could provide helpful contextual information for users to help them reflect on their PA levels (Huang et al., 2016). Although some of reviewed visualisations support calendar view of PA data (e.g. the calendar view on Garmin dashboard that presents training logs), these calendars are standalone and do not integrate with personal calendars to show related data, such as events or work activities.

\subsubsection{Supporting Multiple Types of Visualization}

Providing different choices of visualisations is important to enable the users to customize their experience (Fan et al., 2012). Moreover, different visualisation designs afford different perspectives and understanding of the data, therefore it may address the challenge of the diversity of users' requirements and needs (Tong et al., 2015).

Health data, such as sleep, are represented differently to the activity data on our reviewed devices. Fitbit, Garmin and Samsung use heat maps to encode sleep quality by showing the minutes of deep and light sleep against minutes of wakefulness (e.g. Figure 9), and heat maps of several days could be viewed as bar charts to compare one night's sleep with a past night's sleep. HR visualisation on Fitbit app for a single exercise (presented in (Fitbit, n.d.-a)) gives two types of presentations for HR details: a coloured bar chart and a coloured line chart. The different charts convey different information about one data variable (i.e. HR). As illustrated in Table 4, long-term data are mainly presented in one type of visualisation, and mostly, it is either through bar chart or line chart with the aid of some textual and numerical annotation. However, there is a significant limitation in supporting different methods of visualisation for the different data types.

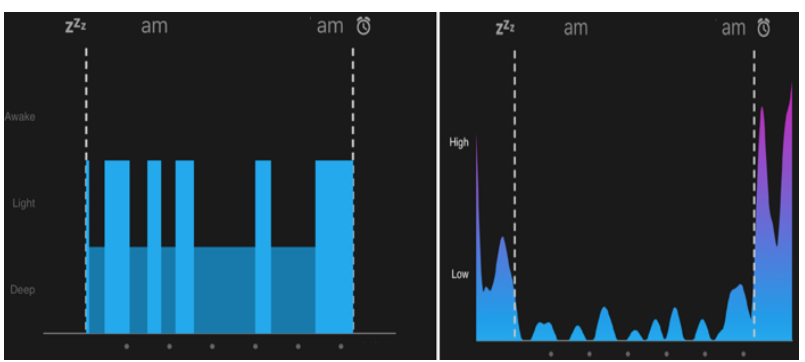

Figure 9 Two screenshots of different sleep visualization on Garmin app.

\subsection{Data Resolution}

A major challenge in designing health and PA visualisation is how many days of data should be represented, especially since the data size grows massively over time.

Rooksby et al. (2014), interviewed 22 participants, who used different activity trackers to understand users' behaviour in self-tracking. Their findings showed that participants were mainly interested in short term data i.e. usually the data collected on the same day only. This indicates that users were interested in meeting their daily goals. In such cases, the view of daily progress on the device screen should be satisfactory for most users (e.g. circular ring). Rooksby et al. also identified five different types of personal tracking based on the participants' tracking purposes: directive, documentary, diagnostic, collecting rewards and fetishised tracking (i.e. tracking for technology and data collection purposes). Their findings showed that there were some participants who were interested in historical data views and would have liked to perform further analysis on the data (e.g. finding a relationship between activity data and sleep quality). Participants who valued long-term tracking had collected data for many years.

In our survey, we asked participants how frequently they checked different types of tracked data (e.g. step count, distance and sleep) to identify the length of visualisation they were interested in, for example, daily or weekly. Participants' responses showed that those who were interested in the aforementioned data were more likely to check their progress towards a target daily. This finding highlights the 
importance of providing a detailed view of PA data on a daily basis.

The visualisation of data on our shortlisted devices was generally for the current day's data or for single exercises performed on that day. Fitbit Blaze and Apple watch visualise activity data for the current day only. On the other hand, Garmin Forerunner 935 and Samsung Gear S3 represent some activity data for more than one day (e.g. bar chart for step count for one week).

\subsection{Sharing Health Data}

The devices we have reviewed in this article have limitations in sharing visualised health and activity data. They either allow sharing the data in textual form (e.g. sharing a summary of an activity with Activity app with non-apple watch users) or restricting visualisation sharing to users added in the social platform of the device dashboard (e.g. Fitbit and Garmin Dashboards). The latter allows users to compare their activity levels with others. However, Fitbit app allows sharing the visualisation of a specific day (as presented in Figure 2) and it allows users to perform simple customization by changing the background colour or choosing the background picture according to users' preferences. Moreover, the user has limited control over resolution and dimensionality of the data to be shared.

Personal data is sensitive, and while sharing PA data could be motivating and could lead to behaviour change, it could also reveal a significant amount of information to others and compromise the privacy of the user (Huang et al., 2015). Sharing features provided by our shortlisted apps and devices are generally for social purposes, for example, for competing with friends. However, sharing activity and health data representations could have broader benefits, such as for medical purposes (if shared with a GP or other healthcare providers), or research related to public health (e.g. long-term effects of medications on general health and wellbeing) or friends and family (who are carers and/or live far away).

The companion apps of devices reviewed in this research do not provide abstract visual encoding of the data. The supported visualisations, such as statistical charts with textual and numerical details, are clear and could reveal high levels of information about oneself, especially when sharing a significant amount of data (e.g. publicly posting daily PA details). These visualisation options are not suitable for sharing when privacy is a concern due to the sensitivity of personal health data. There are a number of abstract visualisations that could be applied to maintain users' privacy for sharing purposes, such as UbiFit (Consolvo et al., 2008) and Spark (Fan et al., 2012), as suggested by Huang et al. (2015). Another consideration is the need to be aware of the possible negative consequences that could result from sharing health and activity data, such as stress, guilt or perceived failure (Huang et al., 2015).

\section{CONCLUSION}

In this article, we have presented an up-to-date review of the visualisations of health and physical activity data tracked by four of the most recent and popular tracking devices on the market. Our review suggests that future personal health systems need to address the following issues:

- There is a gap in terms of the visualisation methods combining different aspects of health data. For example, it could be helpful for some users to see how their sleep (total hours and quality) affects their daily physical activity or vice versa.

- Discussed visualisation methods were essentially designed for data tracked by one device. There are many users who have access to multiple self-tracking devices and, as a result, they face significant limitations in visualising all tracked data on one platform (app/dashboard). Ideally, all devices and apps should integrate with each other and across multiple platforms. Moreover, users could be allowed to select what data to integrate and for how long. This would provide users with better engagement and management of their health data.

- Our review showed that the basic visualisation methods used are statistical charts. Although these methods provide good interpretation of the data, combining them with more advanced methods could provide users with a better understanding.

- There is still a need for adding analytical features and interactive visualisation. For example, the user could filter data being visualised to view only the days where they met their daily goal, to view the level of activity at a specific time on a specific day, or to view the HR details for a specific exercise.

- Visualization methods that offer high personal data encodings are required for social sharing purposes. The devices and apps should offer some abstract representations as an alternative when details of health data are not required.

We hope that this article will help to inspire some novel ideas for visualisation and data representation of health and physical activity within the research of designers who work with emerging and interactive technologies. 


\section{REFERENCES}

Analytical, M. (2016, December). Samsung Gear S3 More Tips \& Tricks - Customise the Menus etc. Retrieved from https://www.youtube.com/watch?v=hDtbyfrex $\mathrm{zQ}$

Apple Inc. (2017). A bold new way to look at your health. Retrieved from https://www.apple.com/uk/ios/health/

Apple Support. (2017, April). Share your Activity with your Apple Watch. Retrieved from https://support.apple.com/en-gb/HT207014

Asimakopoulos, S., Asimakopoulos, G., \& Spillers, F. (2017). Motivation and User Engagement in Fitness Tracking: Heuristics for Mobile Healthcare Wearables. Informatics, 4(1). https://doi.org/10.3390/informatics4010005

Bardram, J. E., \& Frost, M. (2016). The Personal Health Technology Design Space. IEEE Pervasive Computing, 15(2), 70-78. https://doi.org/10.1109/MPRV.2016.37

Borgo, R., Abdul-Rahman, A., Mohamed, F., Grant, P. W., Reppa, I., Floridi, L., \& Chen, M. (2012). An Empirical Study on Using Visual Embellishments in Visualization. IEEE Transactions on Visualization and Computer Graphics, 18(12), 2759-2768. https://doi.org/10.1109/TVCG.2012.197

Business Wire. (2016, January). Fitbit Reveals Fitbit Blaze - The Smart Fitness Watch That Empowers Consumers to Get Fit in Style. Retrieved from http://www.businesswire.com/ news/home/20160105005568/en/

Choe, E. K., Lee, B., \& Schraefel, M. C. (2015). Characterizing Visualization Insights from Quantified Selfers' Personal Data Presentations. IEEE Computer Graphics and Applications, 35(4), 28-37. https://doi.org/10.1109/MCG.2015.51

Choe, E. K., Lee, N. B., Lee, B., Pratt, W., \& Kientz, J. A. (2014). Understanding quantified-selfers' practices in collecting and exploring personal data. In Proceedings of the 32nd annual ACM conference on Human factors in computing systems - CHI '14 (pp. 1143-1152). New York, NY, USA: ACM. https://doi.org/10.1145/2556288.2557372

Consolvo, S., McDonald, D. W., Toscos, T., Chen, M. Y., Froehlich, J., Harrison, B., ... Landay, J. A. (2008). Activity Sensing in the Wild: A Field Trial of Ubifit Garden. In Proceedings of the SIGCHI Conference on Human Factors in Computing Systems (pp. 1797-1806). New York, NY, USA: ACM. https://doi.org/10.1145/1357054.1357335
Cunningham, S. J., \& Jones, M. (2005). Autoethnography: A Tool for Practice and Education. In Proceedings of the 6th ACM SIGCHI New Zealand Chapter's International Conference on Computer-human Interaction: Making CHI Natural (pp. 1-8). New York, NY, USA: ACM.

https://doi.org/10.1145/1073943.1073944

DC RAINMAKER. (2017, March). Garmin Forerunner 935 In-Depth Review. Retrieved from

https://www.dcrainmaker.com/2017/03/garmin -forerunner-935-depth-review.html

Epstein, D., Cordeiro, F., Bales, E., Fogarty, J., \& Munson, S. (2014). Taming Data Complexity in Lifelogs: Exploring Visual Cuts of Personal Informatics Data. In Proceedings of the 2014 Conference on Designing Interactive Systems (pp. 667-676). New York, NY, USA: ACM. https://doi.org/10.1145/2598510.2598558

Fan, C., Forlizzi, J., \& Dey, A. K. (2012). A Spark of Activity: Exploring Informative Art As Visualization for Physical Activity. In Proceedings of the 2012 ACM Conference on Ubiquitous Computing (pp. 81-84). New York, NY, USA: ACM. https://doi.org/10.1145/2370216.2370229

Fitbit. (n.d.-a). Fitbit Blaze Introductory Guide. Retrieved from https://www.fitbit.com/uk/blaze/blaze-101

Fitbit. (n.d.-b). Fitbit Blaze User Manual. Retrieved from https://staticcs.fitbit.com/content/assets/help/ manuals/manual_blaze_en_US.pdf

Fitbit. (n.d.-c). Fitbit YouTube Channel. Retrieved from https://www.youtube.com/user/FitbitOfficialSit e/videos

Fitbit. (2014, October). Fitbit Announces Fitbit Charge, Fitbit Charge HR and Fitbit Surge - 3 New Fitness Trackers for Everyday, Active and Performance Consumers. Retrieved from http://www.businesswire.com/news/home/201 60105005568/en/

Fitbit. (2016a, January). Fitbit Reveals Fitbit Blaze The Smart Fitness Watch That Empowers Consumers to Get Fit in Style. Retrieved from https://investor.fitbit.com/press/pressreleases/press-release-details/2016/FitbitReveals-Fitbit-Blaze--The-Smart-FitnessWatch-That-Empowers-Consumers-to-GetFit-in-Style/default.aspx

Fitbit. (2016b, January). Introducing Fitbit Blaze. Retrieved from https://www.youtube.com/watch?v=3k3DNT5 4NkA\&feature=youtu.be 
Fitbit. (2017). Fitbit App: The fitness app for everyone. Retrieved from https://www.fitbit.com/uk/app

GadFit. (2015, November). ELEVATE YOUR TRAINING WITH GARMIN VIVOSMART HR USER REVIEW | GADFIT. Retrieved from http://www.gadfit.com/garmin-vivosmart-hruser-review/

Garmin. (n.d.-a). Garmin FORERUNNER 935ß)space Owner's Manual. Retrieved from http://static.garmin.com/pumac/Forerunner93 5_OM_EN.pdf

Garmin. (n.d.-b). Garmin YouTube Channel. Retrieved from https://www.youtube.com/ user/garminblog/videos

Garmin. (2009, October). Share, Store and Explore at Garmin Connect. Retrieved from https://www.youtube.com/watch?v=Wsoug4F JwoU

Garmin Press Release. (2017a, March). Garmin introduces the Forerunner 935 - a premium GPS running and triathlon watch. Retrieved from https://www.garmin.com/enGB/blog/garmin-introduces-the-forerunner935-a-premium-gps-running-and-triathlonwatch/

Garmin Press Release. (2017b, April). Introducing the Garmin vivosmart 3, a smart activity tracker with wrist-based heart rate and new fitness, wellness monitoring tools. Retrieved from https://www.garmin.com/enGB/blog/introducing-the-garmin-vivosmart-3a-smart-activity-tracker-with-wrist-basedheart-rate-and-new-fitn/

Gouveia, R., Karapanos, E., \& Hassenzahl, M. (2015). How Do We Engage with Activity Trackers?: A Longitudinal Study of Habito. In Proceedings of the 2015 ACM International Joint Conference on Pervasive and Ubiquitous Computing (pp. 1305-1316). New York, NY, USA: ACM. https://doi.org/10.1145/2750858.2804290

Huang, D., Tory, M., Aseniero, B. A., Bartram, L., Bateman, S., Carpendale, S., ... Woodbury, R. (2015). Personal Visualization and Personal Visual Analytics. IEEE Transactions on Visualization and Computer Graphics, 21(3), 420-433. https://doi.org/10.1109/TVCG.2014.2359887

Huang, D., Tory, M., \& Bartram, L. (2016). A Field Study of On-Calendar Visualizations. In Proceedings of Graphics Interface 2016 (pp. 13-20). Canadian Human-Computer Communications Society / Société canadienne du dialogue humain-machine. https://doi.org/10.20380/GI2016.03
IDC Research, Inc. (2017, March). Wearables Aren't Dead, They're Just Shifting Focus as the Market Grows 16.9\% in the Fourth Quarter, According to IDC. Retrieved from http://www.idc.com/getdoc.jsp?containerld=pr US42342317

Javed, W., McDonnel, B., \& Elmqvist, N. (2010). Graphical Perception of Multiple Time Series. IEEE Transactions on Visualization and Computer Graphics, 16(6), 927-934. https://doi.org/10.1109/TVCG.2010.162

Le, T., Reeder, B., Thompson, H. J., \& Demiris, G. (2013). Health Providers' Perceptions of Novel Approaches to Visualizing Integrated Health Information. Methods of Information in Medicine, 52(3), 250-258.

https://doi.org/10.3414/ME12-01-0073

Li, I., Dey, A., \& Forlizzi, J. (2010). A Stage-based Model of Personal Informatics Systems. In Proceedings of the SIGCHI Conference on Human Factors in Computing Systems (pp. 557-566). New York, NY, USA: ACM. https://doi.org/10.1145/1753326.1753409

Li, I., Dey, A. K., \& Forlizzi, J. (2011). Understanding My Data, Myself: Supporting Self-reflection with Ubicomp Technologies. In Proceedings of the 13th International Conference on Ubiquitous Computing (pp. 405-414). New York, NY, USA: ACM. https://doi.org/10.1145/2030112.2030166

Lin, J. J., Mamykina, L., Lindtner, S., Delajoux, G., \& Strub, H. B. (2006). Fish'N'Steps: Encouraging Physical Activity with an Interactive Computer Game. In Proceedings of the 8th International Conference on Ubiquitous Computing (pp. 261-278). Berlin, Heidelberg: Springer-Verlag. https://doi.org/10.1007/11853565_16

Luke Edwards. (2016, September). Garmin Forerunner 735XT review. Retrieved from https://www.wareable.com/garmin/garminforerunner-735xt-review

Mayer, J., Kazakova, A., \& Boll, S. (2016). Visualization of Complex Health Data on Mobile Devices. In MMHealth 2016 : Multimedia for personal health and health care. New York, NY, USA: ACM.

Michael Sawh. (2017, May). Garmin Forerunner 935 review. Retrieved from https://www.wareable.com/garmin/garminforerunner-935-review

Reseach2Guidance. (2016). mHealth App Developer Economics 2016. Retrieved from https://research2guidance.com/product/mheal th-app-developer-economics-2016/ 
Rooksby, J., Rost, M., Morrison, A., \& Chalmers, M. C. (2014). Personal Tracking As Lived Informatics. In Proceedings of the 32Nd Annual ACM Conference on Human Factors in Computing Systems (pp. 1163-1172). New York, NY, USA: ACM. https://doi.org/10.1145/2556288.2557039

Samsung. (n.d.). Samsung Mobile YouTube Channel. Retrieved from https://www.youtube.com/user/SamsungMobil e/videos

Samsung. (2016, August). Samsung Gear S3: Official Launch Film. Retrieved from https://www.youtube.com/watch?v=ynTB211p $1 \mathrm{PQ}$

Samsung. (2017, April). Samsung User Manual. Retrieved from http://downloadcenter. samsung.com/content/UM/201701/20170117 210121872/SM-R760_R765_R770_UM_EU_ Tizen_Eng_Rev.1.1_170111.pdf

Samsung.com. (2016, September). Samsung Expands Smartwatch Portfolio with Gear S3. Retrieved from https://news.samsung.com/ global/samsung-expands-smartwatchportfolio-with-gear-s3

Samsung Electronics Co., Ltd. (2017). Samsung Health. Retrieved from https://play.google.com/store/apps/details?id= com.sec.android.app.shealth

Samsung Newsroom. (2015, May). Track, Manage, Improve: Better Health with S Health App.

Samsung Newsroom. (2016a, July). New S Health Features Add Fun to Fitness.

Samsung Newsroom. (2016b, August). New S Health Lets Users Team Up to Tone Up.

Samsung Newsroom. (2016c, September). [HandsOn] The Samsung Gear S3. Retrieved from https://news.samsung.com/global/hands-onthe-samsung-gear-s3

Samsung Newsroom. (2017, April). Samsung Releases Comprehensive Gear S3 Value Pack Update.

Shneiderman, B., Plaisant, C., \& Hesse, B. W. (2013). Improving Healthcare with Interactive Visualization. IEEE Computer, 46(5), 58-66. Retrieved from http://dx.doi.org/10.1109/ MC.2013.38;

Tong, X., Gromala, D., Bartram, L., Rajabiyazdi, F., \& Carpendale, S. (2015). Evaluating the Effectiveness of Three Physical Activity Visualizations? How People Perform vs. Perceive. In Electronic proceedings of the IEEE VIS 2015 workshop Personal Visualization: Exploring Data in Everyday Life. Chicago.
TV, T. (2017, April). Every Samsung Gear S3 App, In Depth Review. Retrieved from https://www.youtube.com/watch?v=O-f2mmOFp0 


\section{APPENDICES}

Table 2 Abbreviations used in Table 4.

\begin{tabular}{|l|l|l|l|l|l|}
\hline $\begin{array}{c}\text { Visualization } \\
\text { Technique }\end{array}$ & \multicolumn{1}{|c|}{ Abbreviation } & $\begin{array}{c}\text { Visualization } \\
\text { Technique }\end{array}$ & \multicolumn{1}{|c|}{ Abbreviation } & \multicolumn{1}{|c|}{$\begin{array}{c}\text { Visualization } \\
\text { Technique }\end{array}$} & \multicolumn{1}{|c|}{ Ibreviation } \\
\hline Bar Chart & BC & Line Chart & LC & Animated Icon & Al \\
\hline Circular Ring & CR & Route on Map & RoM & Donut Chart & DC \\
\hline Text \& Numbers & T\&N & Calendar View & CV & Pyramid & PY \\
\hline Heat Map & HM & Clock Metaphor & CM & & \\
\hline
\end{tabular}

Table 3 Colour codings used in Table 4.

\begin{tabular}{|l|l|l|l|l|}
\hline Colour Coding & Weekly & Per Exercise & $\begin{array}{l}\text { Data }>\text { Week } \\
\text { (visualization per day) }\end{array}$ & $\begin{array}{l}\text { Data }>\text { Week } \\
\text { (Day/week/month/year) }\end{array}$ \\
\hline $\begin{array}{l}\text { Daily / Current } \\
\text { represents data of } \\
\text { one day only. }\end{array}$ & $\begin{array}{l}\text { The visualisation } \\
\text { represents data up to } \\
\text { one week. }\end{array}$ & $\begin{array}{l}\text { The } \\
\text { visualisation } \\
\text { represents data } \\
\text { of a single } \\
\text { exercise. }\end{array}$ & $\begin{array}{l}\text { The visualisation } \\
\text { represents data of more } \\
\text { than one week, and it } \\
\text { represents a single } \\
\text { visualisation for each day } \\
\text { in the view. }\end{array}$ & $\begin{array}{l}\text { The visualisation represents } \\
\text { data } \\
\text { it represents all the data in one } \\
\text { visualisation. }\end{array}$ \\
\end{tabular}


Table 4 Main visualisations used on the selected devices and their dashboards / companion phone apps.

\begin{tabular}{|c|c|c|c|c|c|c|c|c|c|c|c|c|c|c|}
\hline $\begin{array}{l}\text { Tracker } \\
\text { Name }\end{array}$ & Platform & $\begin{array}{l}\text { Step } \\
\text { Count }\end{array}$ & Distance & $\begin{array}{l}\text { Flight } \\
\text { Climbed }\end{array}$ & $\begin{array}{l}\text { Heart } \\
\text { Rates }\end{array}$ & $\begin{array}{l}\text { Time in } \\
\text { HR Zones }\end{array}$ & $\begin{array}{l}\text { Active } \\
\text { Minutes }\end{array}$ & $\begin{array}{l}\text { Calories/ } \\
\text { Active } \\
\text { Calories }\end{array}$ & $\begin{array}{l}\text { Exercise/ } \\
\text { Exercise } \\
\text { Minutes }\end{array}$ & $\begin{array}{l}\text { Walking/ } \\
\text { running/ } \\
\text { cycle }\end{array}$ & Pace & $\begin{array}{l}\text { Elevation } \\
\text { Gain }\end{array}$ & $\begin{array}{l}\text { Stand/ } \\
\text { Activity } \\
\text { Hours }\end{array}$ & Sleep \\
\hline \multirow{7}{*}{$\begin{array}{l}\text { Fitbit } \\
\text { Blaze } \\
\text { (No } \\
\text { GPS) }\end{array}$} & \multirow[t]{3}{*}{$\begin{array}{l}\text { Phone } \\
\text { App }\end{array}$} & $\overline{\mathrm{CRR} / \mathrm{BC}}$ & $\begin{array}{l}\mathrm{CR} / \mathrm{BC} / \\
\mathrm{T} \& \mathrm{~N}\end{array}$ & $\begin{array}{l}\mathrm{CR} / \mathrm{BC} / \\
\mathrm{T} \& \mathrm{~N}\end{array}$ & $\begin{array}{l}\mathrm{BC} / \mathrm{LC} / \\
\mathrm{T} \& \mathrm{~N}\end{array}$ & $\begin{array}{l}\mathrm{LC} / \mathrm{BC} / \\
\mathrm{T} \& \mathrm{~N}\end{array}$ & $\begin{array}{l}\mathrm{CR} / \mathrm{BC} / \\
\mathrm{T} \& \mathrm{~N}\end{array}$ & LC/ T\&N & \multirow[t]{3}{*}{ CV } & \multirow[t]{3}{*}{ "RoM/ T\&N } & \multirow[t]{3}{*}{$\begin{array}{l}\mathrm{BC} / \\
\mathrm{T} \& \mathrm{~N}\end{array}$} & \multirow[t]{3}{*}{ LC/ T\&N } & \multirow[t]{3}{*}{ CM/ T\&N } & $\begin{array}{l}\mathrm{BC} / \mathrm{HM} / \\
\mathrm{T} \& \mathrm{~N}\end{array}$ \\
\hline & & $\mathrm{BC} / \mathrm{T} \& \mathrm{~N}$ & $\mathrm{BC} / \mathrm{T} \& \mathrm{~N}$ & $\mathrm{BC} / \mathrm{T} \& \mathrm{~N}$ & $\mathrm{BC} / \mathrm{LC} /$ & LC/BC/ & $\mathrm{BC} / \mathrm{T} \& \mathrm{~N}$ & $\mathrm{CR} / \mathrm{BC} / \mathrm{T} \& \mathrm{~N}$ & & & & & & \multirow{2}{*}{$\begin{array}{l}\mathrm{CM} / \mathrm{HM} / \\
\mathrm{T} \& \mathrm{~N}\end{array}$} \\
\hline & & & & & & & & $\mathrm{BC} / \mathrm{T} \& \mathrm{~N}$ & & & & & & \\
\hline & \multirow[t]{2}{*}{$\begin{array}{l}\text { Dashboa } \\
\text { rd }\end{array}$} & $\begin{array}{l}\mathrm{CR} / \mathrm{BC} / \\
\mathrm{T} \& \mathrm{~N}\end{array}$ & $\begin{array}{l}\mathrm{CR} / \mathrm{BC} / \\
\mathrm{T} \& \mathrm{~N}\end{array}$ & $\begin{array}{l}\mathrm{CR} / \mathrm{BC} / \\
\mathrm{T} \& \mathrm{~N}\end{array}$ & $\begin{array}{l}\mathrm{BC} / \mathrm{LC} / \\
\mathrm{T} \& \mathrm{~N}\end{array}$ & $\begin{array}{l}\mathrm{LC} / \mathrm{BC} / \\
\mathrm{T} \& \mathrm{~N}\end{array}$ & $\begin{array}{l}\mathrm{CR} / \mathrm{BC} / \\
\mathrm{T} \& \mathrm{~N}\end{array}$ & $\mathrm{LC} / \mathrm{T} \& \mathrm{~N}$ & \multirow[t]{2}{*}{ CV } & \multirow[t]{2}{*}{ RoM/ T\&N } & \multirow[t]{2}{*}{$\begin{array}{l}\mathrm{BC} / \\
\mathrm{T} \& \mathrm{~N}\end{array}$} & \multirow[t]{2}{*}{ LC/T\&N } & \multirow[t]{2}{*}{$\mathrm{CM} / \mathrm{T} \& \mathrm{~N}$} & $\begin{array}{l}\mathrm{BC} / \mathrm{HM} / \\
\mathrm{T} \& \mathrm{~N}\end{array}$ \\
\hline & & $\mathrm{BC} / \mathrm{T} \& \mathrm{~N}$ & $\mathrm{BC} / \mathrm{T} \& \mathrm{~N}$ & $\mathrm{BC} / \mathrm{T} \& \mathrm{~N}$ & $\begin{array}{l}\mathrm{BC} / \mathrm{LC} / \\
\mathrm{T} \& \mathrm{~N}\end{array}$ & $\begin{array}{l}\text { LC/BC/ } \\
\mathrm{T} \& \mathrm{~N}\end{array}$ & $\mathrm{BC} / \mathrm{T} \& \mathrm{~N}$ & $\begin{array}{l}\mathrm{CR} / \mathrm{BC} / \mathrm{T} \& \mathrm{~N} \\
\mathrm{BC} / \mathrm{T} \& \mathrm{~N}\end{array}$ & & & & & & $\begin{array}{l}\mathrm{CM} / \mathrm{HM} / \\
\mathrm{T} \& \mathrm{~N}\end{array}$ \\
\hline & \multirow{2}{*}{$\begin{array}{l}\text { Device } \\
\text { Screen }\end{array}$} & $\mathrm{CR} / \mathrm{T} \& \mathrm{~N}$ & $\mathrm{CR} / \mathrm{T} \& \mathrm{~N}$ & $\mathrm{CR} / \mathrm{T} \& \mathrm{~N}$ & $\mathrm{~T} \& \mathrm{~N} / \mathrm{Al}$ & \multirow[t]{2}{*}{$\mathrm{T} \& \mathrm{~N} / \mathrm{Al}$} & $\mathrm{CR} / \mathrm{T} \& \mathrm{~N}$ & $\mathrm{CR} / \mathrm{T} \& \mathrm{~N}$ & \multirow[t]{2}{*}{$T \& N$} & \multirow[t]{2}{*}{ T\&N } & \multirow[t]{2}{*}{ T\&N } & \multirow{2}{*}{ - } & \multirow[t]{2}{*}{ T\&N } & \multirow[t]{2}{*}{$\mathrm{CR}$} \\
\hline & & $\mathrm{T} \& \mathrm{~N}$ & $\mathrm{~T} \& \mathrm{~N}$ & $\mathrm{~T} \& \mathrm{~N}$ & $\mathrm{~T} \& \mathrm{~N}$ & & $\mathrm{~T} \& \mathrm{~N}$ & $\mathrm{~T} \& \mathrm{~N}$ & & & & & & \\
\hline \multirow{3}{*}{$\begin{array}{l}\text { Apple } \\
\text { Watch } \\
\text { (Activity } \\
\text { App) }\end{array}$} & \multirow{2}{*}{$\begin{array}{l}\text { Phone } \\
\text { App }\end{array}$} & $\mathrm{T} \& \mathrm{~N}$ & $\mathrm{~T} \& \mathrm{~N}$ & \multirow[b]{2}{*}{ - } & $\mathrm{T} \& \mathrm{~N}$ & \multirow[b]{2}{*}{ - } & \multirow{2}{*}{$\mathrm{T} \& \mathrm{~N}$} & $\begin{array}{l}\mathrm{BC} / \mathrm{CR}+ \\
\mathrm{T} \& \mathrm{~N}\end{array}$ & $\begin{array}{l}\mathrm{BC} / \mathrm{CR} / \\
\mathrm{T} \& \mathrm{~N}\end{array}$ & \multirow{2}{*}{ RoM/ T\&N } & \multirow{2}{*}{$T \& N$} & \multirow{2}{*}{$T \& N$} & $\begin{array}{l}\mathrm{BC} / \mathrm{CR} \\
\mathrm{T} \& \mathrm{~N}\end{array}$ & \multirow[b]{2}{*}{ - } \\
\hline & & $T \& N$ & T\&N & & $T \& N$ & & & $\mathrm{CV}+\mathrm{CR}$ & $C V+C R$ & & & & $C V+C R$ & \\
\hline & $\begin{array}{l}\text { Device } \\
\text { Screen }\end{array}$ & $\mathrm{T} \& \mathrm{~N}$ & $\mathrm{~T} \& \mathrm{~N}$ & - & $\mathrm{T} \& \mathrm{~N} / \mathrm{Al}$ & - & $T \& N$ & $\begin{array}{l}\mathrm{CR}+\mathrm{BC}+ \\
\mathrm{T} \& \mathrm{~N}\end{array}$ & T\&N & $\mathrm{T} \& \mathrm{~N}$ & $T \& N$ & $\mathrm{~T} \& \mathrm{~N}$ & $\begin{array}{l}\mathrm{BC} / \mathrm{CR} \\
\mathrm{T} \& \mathrm{~N}\end{array}$ & - \\
\hline Health & Phone & $\mathrm{BC}$ & $\mathrm{BC}$ & $\mathrm{BC}$ & $\mathrm{BC}$ & - & - & $\mathrm{BC}$ & $\mathrm{BC}$ & $\mathrm{BC}$ & - & & $\mathrm{BC}$ & $\mathrm{BC}$ \\
\hline app (iOS) & & $\mathrm{T} \& \mathrm{~N}$ & $\mathrm{~T} \& \mathrm{~N}$ & $\mathrm{~T} \& \mathrm{~N}$ & $\mathrm{~T} \& \mathrm{~N}$ & & & $\mathrm{T \& N}$ & $\mathrm{T} \& \mathrm{~N}$ & $\mathrm{~T} \& \mathrm{~N}$ & & & $\mathrm{~T} \& \mathrm{~N}$ & $\mathrm{~T} \& \mathrm{~N}$ \\
\hline Garmin & $\begin{array}{l}\text { Dashboa } \\
\text { rd /App }\end{array}$ & $\begin{array}{l}\mathrm{CR} / \mathrm{BC} / \\
\mathrm{T} \& \mathrm{~N}\end{array}$ & LC/ T\&N & T\&N & LC/T\&N/I & "LC/T\&N/I & $\overline{\mathrm{BCC} / \mathrm{T} \& \mathrm{~N}}$ & T\&N & T\&N & RoM/ T\&N & $\begin{array}{l}\mathrm{LC} / \\
\mathrm{T} \& \mathrm{~N}\end{array}$ & LC/ T\&N & - & $\begin{array}{l}\mathrm{CR} / \mathrm{LC} / \mathrm{HM} / \\
\mathrm{T} \& \mathrm{~N}\end{array}$ \\
\hline & & $\mathrm{BC} / \mathrm{T} \& \mathrm{~N}$ & & $\mathrm{BC} / \mathrm{T} \& \mathrm{~N}$ & $\begin{array}{l}\mathrm{LC} / \mathrm{T} \& \mathrm{~N} \\
\mathrm{LC} / \mathrm{T} \& \mathrm{~N}\end{array}$ & $\mathrm{BC} / \mathrm{T} \& \mathrm{~N}$ & $T \& N$ & T\&N & CV/ T\&N & & & & - & $\begin{array}{l}\mathrm{BC} / \mathrm{HM} / \\
\mathrm{T} \& \mathrm{~N}\end{array}$ \\
\hline & Device & $\mathrm{CR} / \mathrm{T} \& \mathrm{~N}$ & $T \& N$ & $\mathrm{T \& N}$ & $\mathrm{BC} / \mathrm{T} \& \mathrm{~N}$ & $\mathrm{BC} / \mathrm{T} \& \mathrm{~N}$ & T\&N & T\&N & T\&N & RoM/ T\&N & T\&N & $\mathrm{LC} / \mathrm{T} \& \mathrm{~N}$ & — & . \\
\hline & Screen & $\mathrm{BC} / \mathrm{T} \& \mathrm{~N}$ & $\mathrm{BC} / \mathrm{T} \& \mathrm{~N}$ & & LC/T\&N & & $T \& N$ & & & & & & & \\
\hline & App/Das & $\overline{B C} / \mathrm{T} \& \mathrm{~N}$ & T\&N & "BC/ T\&N & T\&N & & & $\mathrm{BC} / \mathrm{T} \& \mathrm{~N}$ & & T\&N & & T\&N & T\&N & \\
\hline & hboard & DU II QIV & & & LC/ T\&N & - & $\mathrm{T} \& \mathrm{~N}$ & & T\&N & & T\&N & & $1 \& I N$ & T\&N \\
\hline Samsung & & $\mathrm{BC} / \mathrm{T} \& \mathrm{~N}$ & $\mathrm{~T} \& \mathrm{~N}$ & $\mathrm{BC} / \mathrm{T} \& \mathrm{~N}$ & LC/ T\&N & - & & $T \& N$ & & RoM/T\&N & & & & $\mathrm{BC} / \mathrm{T} \& \mathrm{~N}$ \\
\hline $\begin{array}{l}\text { (Gear } \\
\text { S3) }\end{array}$ & $\begin{array}{l}\text { Device } \\
\text { Screen }\end{array}$ & $\begin{array}{l}\mathrm{DC} / \mathrm{BC} / \\
\mathrm{CR} / \mathrm{PY} / \\
\mathrm{T} \& \mathrm{~N}\end{array}$ & CR/ T\&N & $\mathrm{DC} / \mathrm{T} \& \mathrm{~N}$ & $\mathrm{LC} / \mathrm{T} \& \mathrm{~N}$ & $\mathrm{BC} / \mathrm{T} \& \mathrm{~N}$ & $T \& N$ & $\begin{array}{l}\mathrm{CM} / \mathrm{DC} / \mathrm{T} \& \mathrm{~N} \\
\mathrm{~T} \& \mathrm{~N}\end{array}$ & $\mathrm{DC} / \mathrm{T} \& \mathrm{~N}$ & RoM/T\& N & $\begin{array}{l}\mathrm{LC} / \\
\mathrm{T} \& \mathrm{~N}\end{array}$ & LC/T\&N & T\&N & $\mathrm{CM} / \mathrm{HM} / \mathrm{T} \& \mathrm{~N}$ \\
\hline & & $\mathrm{BC} / \mathrm{T} \& \mathrm{~N}$ & & & & & & & T\&N & & & & & \\
\hline
\end{tabular}

